\title{
Há uma relação específica entre Consciência Morfológica e Reconhecimento de Palavras?
}

\author{
Luciene Corrêa Miranda - Universidade Federal de Juiz de Fora, Juiz de Fora/ MG, Brasil \\ Márcia Maria Peruzzi Elia da Mota - Universidade Salgado de Oliveira, Juiz de Fora/MG, Brasil
}

\begin{abstract}
Resumo
Estudos realizados em línguas alfabéticas mostram que a consciência morfológica - habilidade de refletir sobre os morfemas que compõem as palavras - está associada ao sucesso no reconhecimento e compreensão de palavras na leitura e na escrita. O português é uma língua com uma ortografia que se encontra no meio do espectro quando falamos da transparência nas correspondências entre letra e som. Em línguas transparentes, com correspondência grafema-fonema regulares, a consciência morfológica pode não ter o mesmo impacto na aquisição da escrita. Neste estudo investigamos se o processamento da morfologia derivacional em crianças do ensino fundamental contribui para leitura no português e se essa contribuição é independente da decodificação. Utilizou-se uma tarefa de spooneirismo e outra de subtração de fonemas para acessar a decodificação e o TDE para leitura de palavras. Os resultados mostram que a decodificação é uma habilidade muito importante na leitura e que a habilidade de refletir sobre os morfemas contribui para leitura e que essa contribuição é até certo ponto independente da consciência fonológica.

Palavras-chave: Consciência metalinguística, Consciência morfológica, Decodificação fonológica, Leitura, Alfabetização.
\end{abstract}

\section{Is there a specific connection between Morphological Awareness and Word Recognition?}

\begin{abstract}
Studies carried out in alphabetic languages show that morphological awareness - the ability to reflect upon the word's morphemes - is related to the success in recognizing words and comprehension in reading and spelling. Portuguese is a language located in the middle of transparent letter-sound correspondence rules. In transparent languages, those with regular grapheme-phoneme correspondence rules, morphological awareness may not have the impact in written language acquisition. This study investigated whether derivational morphology processing contributes to reading in Portuguese in primary education and whether this contribution is independent of phonological decoding. A phoneme spooneirism task and another of phoneme subtraction task was used to access reading decoding and the TDE was used to assess reading recognition. The results show that decoding is a very important ability to learn to read and that the ability to reflect upon the morphemes of the words contributes to reading and that this contribution is, to a certain extent independent of phonological aw areness.

Key-words: Metalinguistic aw areness, Morphological aw areness, Phonological decoding, Reading, Literacy.
\end{abstract}

\section{¿Hay una relación específica entre consciencia morfológica y reconocimiento de palabras?}

\begin{abstract}
Resumen
Estudios realizados en lenguas alfabéticas señalan que la consciencia morfológica - la habilidad de reflexionar sobre los morfemas que componen las palabras - está asociada al éxito en el reconocimiento y comprensión de palabras tanto en la lectura como en la escritura. El portugués es una lengua con una ortografía que se encuentra a medio camino del espectro en lo que se refiere a la transparencia en términos de la correspondencia entre letras y sonidos. En lenguas transparentes, que tienen una correspondencia grafema-fonema regular, la conciencia morfológica puede no tener el mismo impacto en la adquisición de la escritura. En este estudio investigamos si el procesamien to de la morfología derivativa en niños de Enseñanza Básica contribuye para la lectura en lengua portuguesa, y si dicha contribución es independiente de la descodificación. Se utilizó una tarea de spoonerismo y otra de sustracción de fonemas para acceder a la descodificación además del TDE para la lectura de palabras. Los resultados señalan que la descodificación es una habilidad muy importante en lo que se refiere a la lectura y que la habilidad de reflexionar sobre los morfemas contribuye a la lectura además que dicha contribución es en cierta medida independiente de la consciencia fonológica.

Palabras clave: Consciencia metalingüística, Consciencia morfológica, Decodificación fonológica, Lectura, Alfabetización.
\end{abstract}

Embora usualmente a língua escrita seja analisada no nível da fonologia, a escrita pode ser analisada em vários níveis. Esses níveis envolvem o processamento fonológico e morfológico, entre outros. Marec-Breton e Gombert (2004) salientam que os dois níveis particularmente importantes para alfabetização são os níveis morfológico e fonológico. Os autores descrevem esses níveis como princípios: fonográfico e semiográfico. O primeiro envolve estabelecer como unidades gráficas os grafemas ou letras que correspondem aos sons da fala, como os fonemas ou silabas. O segundo princípio, o semiográfico, envolve estabelecer como os grafemas representam significados. O processamento morfológico está mais fortemente associado ao princípio semiográfico, ao passo que o processamento fonológico está mais fortemente associado ao princípio fonográfico. Morfemas são as menores unidades linguísticas que têm significado próprio. A habilidade de refletir e manipular intencionalmente essas unidades de significado é chamada de consciência morfológica e pode ajudar o aprendiz a entender o princípio semiográfico (Carlisle, 1995). 
O processamento dos morfemas das palavras pode ser importante para leitura porque podem ajudar as crianças a identificar palavras novas com ortografia complexa. Essas palavras aumentam de frequência com a escolarização. Palavras como "açucarado", que têm ortografia ambígua, podem ser escritas de forma correta se soubermos sua origem: "açúcar". Os significados dessas palavras podem ser inferidos também na leitura, se nós soubermos o significado da palavra que as originou. Além da leitura de palavras, é possível que a consciência morfológica ajude na compreensão de texto, para que os conhecimentos sintático/semânticos ligados a esse processamento sejam generalizados para a compreensão das palavras no contexto (Tong e colaboradores, 2011).

Assim, no caso específico da leitura de palavras, podemos pensar que a consciência morfológica pode vir a ajudar na identificação daquelas palavras que não são reconhecidas pela decodificação, como as menos frequentes e que são morfologicamente complexas. O reconhecimento dessas palavras pode vir a ajudar a compreensão de textos, bem como a consciência morfológica pode contribuir de forma direta para compreensão de texto via conhecimento sintático semântico.

De fato, a relação entre a consciência morfológica e a leitura em crianças falantes do inglês foi demonstrada por Joanne Carlisle, que nos seus estudos mostrou que a habilidade de refletir sobre os morfemas das palavras estava associada ao desempenho no reconhecimento de palavras e à compreensão de leitura (Carlisle, 1995, 2000; Carlisle \& Fleming, 2003). Além dos estudos de Carlisle, outras pesquisas realizadas com falantes do inglês obtiveram resultados semelhantes (Deacon \& Kirby, 2004; Nagy, Berninger \& Abbot, 2006).

Não é só no inglês que se tem observado um efeito facilitador da estrutura morfológica no desenvolvimento da leitura. Estudos realizados em outras línguas alfabéticas têm demonstrado que a consciência morfológica contribui para leitura e escrita em diferentes ortografias (Colé, Marec-Breton, Royer \& Gombert; 2003; Sénéchal, Basque \& Leclaire, 2006, em francês; Mota, 2011; em português; e Verhoeven, Schreuder \& Haarman, 2006, em holandês).

No português, Mota, Annibal e Lima (2008) mostraram que a consciência morfológica contribui para o processamento da leitura e escrita. Utilizando regressão múltipla para analisar os dados, investigaram se havia uma relação específica entre a consciência morfológica e a escrita. Isto é, verificaram que os escores da consciência morfológica continuavam a contribuir para a escrita depois de se controlar o processamento fonológico. Porém, as autoras não puderam demonstrar a especificidade dessa relação na leitura, pois não houve correlações significativas entre as tarefas de consciência fonológica e a leitura. Em outro estudo, Mota e colaboradores (2012) investigaram a relação entre a consciência morfológica e a compreensão de texto. Os autores demonstraram a relação entre a consciência morfológica e a compreensão de texto, mas não exploram a relação entre a consciência morfológica e leitura de palavras isoladas, isto é, se a associação encontrada era mediada pelo conhecimento de palavras escritas pelas crianças.

Uma questão teórica que precisa ser mais bem explorada empiricamente é se a consciência morfológica contribui para a leitura de palavras no português. É possível que a relação entre a consciência morfológica e a compreensão de leitura seja mediada pela leitura de palavras. Por outro lado, essa relação pode ser direta. Os conhecimentos sintáticos/semânticos necessários à morfologia podem ajudar na leitura contextual. Se a consciência morfológica ajuda no reconhecimento das palavras de forma direta, isto é, independente, podemos esperar que os escores em tarefas de consciência morfológica contribuam para leitura, mesmo depois de se controlar a contribuição da decodificação fonológica (o conhecimento das relações letra e som).

Há poucos estudos brasileiros sobre a relação entre a consciência morfológica e a língua escrita. No entanto, em outro estudo com crianças do primeiro ciclo do ensino fundamental, Mota (2011) realizou uma correlação parcial entre a medida de analogia gramatical e a leitura, controlando-se, para a variância compartilhada, pela memória de dígitos. A medida de dígitos é também uma medida de processamento fonológico. O resultado, para o primeiro ano, mostra que a consciência morfológica não apresentou correlações significativas, quando dígito e memória são controlados. No entanto, para o segundo ano, o resultado mostrou que a associação entre a consciência morfológica e a leitura foi significativa, mesmo que se controle a memória e vocabulário das crianças.

O objetivo do presente estudo foi explorar a relação entre a consciência morfológica e o reconhecimento de palavras de forma mais específica. Para testar a hipótese de que a consciência morfológica contribui de forma independente para o reconhecimento de palavras, optou-se por controlar o conhecimento das correspondências entre letra e som usando um teste de spooneirismo.

\section{Participantes}

\section{Método}

Participaram da pesquisa 57 alunos: 24 do segundo ano (média de idade 95,7 meses ou 7 ano e 11 
meses, $\mathrm{DP}=5,16)$ e 33 do terceiro ano do ensino fundamental (média de idade 108,1 meses ou 9 anos, $\mathrm{DP}=4,29)$ matriculados em uma escola federal da zona urbana de Juiz de Fora. A média de idade de toda a amostra foi de 102,8 meses ou 8 anos e 6 meses (DP=7,70). O critério de inclusão na pesquisa foi a assinatura de um termo de consentimento livre e esclarecido (TCLE), o qual foi submetido ao Comitê de Ética e Pesquisa (CEP) da UFJF. Participaram apenas as crianças que concordaram com a participação e obtiveram autorização escrita no TCLE.

\section{Instrumentos}

Tarefas de conhecimento das correspondências letra som

Nessas tarefas utilizaram as letras e não os fones, assim mediam o conhecimento da relação entre as letras e os sons.

\section{1- Spoonerismo}

"Spoonerism" é um termo referente aos lapsos da fala, ao nível de morfemas ou fonemas, quando, por exemplo, a pessoa, ao falar uma frase de duas palavras troca a letra inicial da primeira pela letra inicial da segunda (Sternberg, 2000).

Utilizamos a tarefa de Cardoso-Martins (comunicação pessoal de 14 de agosto de 2008), que visou investigar a capacidade da criança de reconhecer fonemas e realizar trocas e manipulações a partir dos mesmos, formando novas palavras ou pseudopalavras. Por exemplo, é perguntado à criança como ficaria a palavrinha "galo" se o seu primeiro som/letra /g/ fosse trocado pelo som de $/ \mathrm{k} /$. O total possível de pontos era de dez em cada parte.

\section{2- Subtração de fonema}

É tarefa originalmente elaborada por D. J. Bruce (1964) em língua inglesa. Utilizamos a versão brasileira adaptada por Cardoso-Martins e Frith (1999). Bruce realizava a leitura de algumas palavras para as crianças e solicitava que imaginassem como aquelas palavras ficariam se algum som fosse retirado das mesmas. (Exemplo: "left" sem o /t/). Ele observou que as crianças de faixa etária igual ou maior que oito anos obtiveram maior êxito na tarefa se comparadas às crianças menores (citado por Bryant \& Bradley, 1987).

Esse tipo de tarefa tem como objetivo verificar a capacidade da criança de manipular os fonemas das palavras, sendo que na tarefa adaptada em língua portuguesa a criança deve retirar os fonemas iniciais de algumas palavras, formando, assim, novas palavras (exemplo: "imundo" sem o som de /i/) ou pseudopalavras (exemplo: "pasta" sem o som de /p/). O total possível de pontos era de dez em cada parte.
Desempenho escolar - TDE

Foram utilizados os itens de leitura de palavras isoladas e os itens de escrita do Teste de Desempenho Escolar - TDE (Stein, 1994). Por meio desse instrumento padronizado, que apresenta propriedades psicométricas satisfatórias, incluindo evidências de validade de critério e bom índice de consistência interna $(\alpha>0,70)$, foi avaliado o desempenho na leitura e escrita das crianças. O total possível de pontos era 70 para o subteste de leitura e 34 para o subteste de escrita.

Inteligência - Teste WISC (Subteste Dígitos)

O subteste de Dígitos da Escala de Inteligência Wechsler para crianças - WISC III (Weschler, 1991) foi utilizado. A habilidade de armazenar informação fonológica na memória foi avaliada pelo subteste de Dígitos do Wechsler Intelligence Scale for Children (WISC), que requer a repetição de sequências de números em sua ordem direta ou inversa. $O$ subteste foi aplicado segundo as instruções expressas em seus manuais. $\mathrm{O}$ coeficientes de fidedignidade para esse subteste foi calculado pelo método de Guttmam, apresentando bons índices de consistência interna (coeficientes maiores que 0,60 ).

Tarefas de consciência morfológica: analogia gramatical

A tarefa inicial de Nunes, Bindman e Bryant (1997) foi adaptada do inglês para o português considerando a especificidade $\mathrm{da}$ morfologia derivacional do português. A tarefa avalia a habilidade da criança em manipular morfemas (Deacon \& Kirby, 2004). Sob essa perspectiva, foram criados dez itens, a partir dos quais a criança devia produzir uma palavra morfologicamente complexa a partir de uma palavraalvo, aplicando a mesma relação de derivação de um par previamente dado (exemplo: pedra-pedreiro; leite?).

\section{Procedimento}

As crianças foram submetidas às tarefas na própria escola onde estavam matriculadas. A aplicação das tarefas se deu em sessões individuais, que se realizaram da seguinte forma: o subteste do TDE de leitura do TDE e as tarefas de analogia gramatical, subtração de fonema e spoonerismo foram aplicadas individualmente nessa ordem. $\mathrm{Na}$ última sessão foi aplicado o subteste do WISC.

$\mathrm{Na}$ primeira parte da tarefa de spoonerismo, perguntou-se à criança, por exemplo: "Eu tenho a palavrinha 'gato'. Como esta palavrinha ficaria se eu trocasse o som de (fonema) /g/ pelo som de (fonema) $/ \mathrm{r} /$ ?". Foram mostrados para a criança três itens de exemplo e a partir daí era esperado que ela realizasse a 
tarefa sozinha. $\mathrm{Na}$ segunda parte da tarefa, continuouse com a instrução: "Agora eu tenho duas palavrinhas, como 'milho verde'. Se eu trocar o som (fonema) da primeira letrinha da primeira palavra com o som (fonema) da primeira letrinha da segunda palavra, como ficaria a nova palavra?" Esperava-se que a criança verbalizasse "vilho merde". A realização da tarefa originou palavras e pseudopalavras e contou apenas com a verbalização da criança, não contendo nenhuma tarefa escrita.

Na tarefa de Subtração de Fonema, uma palavra, como "anão" foi lida para a criança. Em seguida, realizou-se o inquérito: "Tenho aqui a palavrinha 'anão'. Se eu tirar o som da letra (fonema) /a/, como ela ficaria?". Foi esperado que a criança respondesse "não". Foram mostrados para a criança três itens de exemplo e a partir daí era esperado que ela realizasse a tarefa sozinha. As palavras utilizadas, após a subtração do fonema originaram outras palavras ou pseudopalavras. Assim como na tarefa anterior, esta também contou apenas com a verbalização por parte da criança. As aplicações e a correção dos testes WISC e TDE corresponderam às instruções expressas no manual de aplicação de cada teste padronizado.

\section{Resultados}

A Tabela 1 mostra a média e o desvio padrão para o número de acertos em cada tarefa de consciência morfológica e conhecimento das correspondências entre letra e som por ano escolar. A primeira pergunta que levantamos é se há uma relação entre a consciência morfológica, conhecimento das correspondências entre letra e som e o aprendizado da leitura em português. Essa questão é importante para a nossa hipótese, porque, se as correlações não forem significativas não há razão para continuar se explorando a questão. Para responder a essa pergunta, o número de respostas corretas nos testes foi computado e correlações entre as diversas medidas foram efetuadas. Correlações de Pearson foram empregadas.

Tabela 1. Média e o desvio padrão (DP) para o número de respostas corretas para tarefas de consciência morfológica e consciência fonológica por série

\begin{tabular}{lcc}
\hline \multicolumn{1}{c}{ Tarefa } & $2^{\circ}$ Ano & $3^{\circ}$ Ano \\
& Média $(D P)$ & Média $(D P)$ \\
\hline Analogia gramatical & $6,2(1,9)$ & $7,3(1,3)$ \\
Spoonerismo p. 1 & $7,9(2,3)$ & $8,9(1.2)$ \\
Spoonerismo p. 2 & $3,5(4,4)$ & $5,8(3,7)$ \\
Subtração de Fonema p.1 & $9,3(0,9)$ & $9,4(0,8)$ \\
Subtração de Fonema p.2 & $9,0(1,2)$ & $9,3(0,7)$ \\
\hline
\end{tabular}

Os resultados mostraram uma correlação positiva e significativa para a medida de consciência morfológica e leitura $(r=0,38 ; p<0,004)$. Duas das tarefas de conhecimento das correspondências entre letra e som correlacionaram-se de forma positiva e significativa com a leitura: para Spoonerismo parte 1

Tabela 2. Coeficientes de Correlações de Pearson

\begin{tabular}{lcccccc}
\multicolumn{1}{c}{ Variáveis } & 2 & 3 & 4 & 5 & 6 & 7 \\
\hline 1. TDE Leitura & $0,38^{* *}$ & $0,44^{* *}$ & $0,55^{* *}$ & 0,19 & 0,25 & 0,26 \\
2. Analogia Gramatical & & $0,45^{* *}$ & $0,43^{* *}$ & $-0,07$ & 0,16 & 0,13 \\
3. Spoonerismo p.1 & & $0,49^{* *}$ & 0,14 & $0,31^{*}$ & 0,23 \\
4. Spoonerismo p.2 & & & 0,25 & $0,37^{* *}$ & $0,28^{*}$ \\
5. Subtração fonema p.1 & & & & $0,42^{* *}$ & 0,20 \\
6.Subtração fonema p.2 & & & & & 0,16 \\
7.WISC Dígitos & & & & &
\end{tabular}

A segunda pergunta foi se a contribuição da consciência morfológica para a leitura continuava significativa quando a variância referente ao $(r=0,44 ; p<0,001)$; para Spoonerismo parte $2(r=0,55$; $p<0,001)$; para Subtração de fonema parte $1(r=0,19$; $p>0,15)$; para Subtração de Fonema parte $2(\mathrm{r}=0,25$; $\mathrm{p}>0,063)$ os resultados não foram significativos. A Tabela 2 mostra os resultados dessas correlações. conhecimento das correspondências entre letra e som era controlada.

Assim, análises de regressão múltipla com ordem fixa foram realizadas para testar a contribuição da 
tarefa de analogia gramatical para leitura, após se controlarem os efeitos da escolaridade e uma das medidas de conhecimento das correspondências entre letra e som que foi significativa nas correlações realizadas. Os resultados mostraram que a tarefa de analogia gramatical contribui de forma significativa para leitura quando controlados os efeitos da subtração de fonemas parte 1 e parte 2 , primeira medida de avaliação do conhecimento das correspondências entre letra e som, respectivamente $(\beta=0,30 ; p=0,02$ para parte 1 e $\beta=0,27 ; p=0,04$ para parte 2). Essas medidas se correlacionaram de forma estatisticamente significativa com a leitura e consciência morfológica, quando se utiliza uma medida de processamento fonológico, aqui medido pelos dígitos WISC $-(\boldsymbol{\beta}=0,26$; $p=0,053)$, se considerarmos uma medida menos conservadora de $P$. No entanto, não são significativas quando se controlam as tarefas de spooneirismo. A Tabela 3 mostra as demais estimativas para os modelos de regressão ajustados.

Tabela 3. Resultado das regressões múltiplas para o TDE - leitura

\begin{tabular}{lcccl}
\hline \multicolumn{1}{c}{ Variáveis em cada etapa } & Beta & R2 & F & Sig. \\
\hline 1. Série & & & & \\
2. Spooneirismo parte 1 & 0,38 & 0,26 & 9,51 & $0,003^{* *}$ \\
3. Analogia & 0,16 & 0,28 & 6,86 & 0,24 \\
2. Spooneirismo parte 2 & 0,49 & 0,35 & 14,44 & $0,0001^{* *}$ \\
3. Analogia & 0,12 & 0,36 & 9,89 & 0,35 \\
2. Subtração de fonemas1 & 0,17 & 0,16 & 5,1 & 0,16 \\
3. Analogia & 0,30 & 0,24 & 5,6 & $0,02^{*}$ \\
2. Subtração de fonemas2 & 0,20 & 0,17 & 5,4 & 0,12 \\
3. Analogia & 0,27 & 0,23 & 5,2 & $0,04^{*}$ \\
2. WISC dígitos & 0,27 & 0,18 & 5,7 & $0,03^{*}$ \\
3. Analogia & 0,26 & 0,24 & 5,3 & $0,053^{*}$ \\
\hline *P<0,01/** $p<0,001$ & & & &
\end{tabular}

\section{Discussão}

A literatura na área mostra que a decodificação é um importante aspecto da leitura e da escrita. Esse ponto tem sido demonstrado pelas fortes associações encontradas entre tarefas de consciência fonológica e a leitura em várias línguas alfabéticas, bem como no português (Barrera \& Maluf, 2003; Bradley \& Bryant, 1983; Capovilla \& Capovilla, 2000). Nossos resultados são condizentes com o desses estudos. Não estava no escopo desse estudo replicar esses estudos. A questão investigada foi se a relação entre a consciência morfológica e a leitura de palavras se sustenta quando se controla a variância partilhada com a decodificação. Se essa relação se mostra significativa quando não se controla a decodificação, podemos sugerir que processos lexicais estão em jogo quando a criança reconhece palavras no português. Duas das quatro tarefas que envolviam o conhecimento das correspondências entre letra e som, as tarefas de spooneirismo, se mostraram associadas à leitura, mas a relação entre a consciência morfológica e a leitura não se manteve quando a decodificação foi controlada com uma tarefa mais conservadora como a de spooneirismo.

Em um estudo publicado por Mota, Annibal e Lima (2008), as autoras não detectaram correlações significativas entre as tarefas de consciência fonológica que empregaram e a leitura das crianças de segundo e terceiro ano. As tarefas utilizadas foram a de rima e aliteração que podem ter sido muito fáceis para crianças nessa faixa etária, e, portanto, não ter sido apropriada para medir a consciência fonológica das crianças que, como no presente estudo, já eram alfabetizadas. Tarefas que envolvem a manipulação fonêmica são mais adequadas para essa faixa etária.

Os resultados obtidos pelas correlações parecem indicar que ainda para as crianças mais velhas a decodificação é importante aspecto da leitura de palavras. As crianças que dominaram melhor as tarefas de spooneirismo foram melhores no reconhecimento de palavras. Por outro lado, a habilidade de refletir sobre os morfemas também se apresentou como outra habilidade relacionada ao bom desempenho na leitura de palavras.

Correlações simples não controlam variância partilhada entre as tarefas medidas. Regressões foram utilizadas para controlar a variância partilhada entre variáveis. Os resultados das regressões que, controlaram para série escolar mostram que a tarefa de analogia gramatical contribui para leitura de palavras no português. Isso ocorre mesmo quando se controla também a tarefa de dígitos, que é uma medida de processamento fonológico. No entanto, quando as tarefas de decodificação entram na análise, essa contribuição deixa de ser significativa. 
Esses resultados sugerem que a consciência morfológica tem um papel a cumprir no reconhecimento de palavras. Porém, quando tiramos a variância atribuída ao conhecimento das correspondências entre letra e som medida pela tarefa de spooneirismo, os escores da consciência morfológica deixam de ser significativos. A tarefa de spooneirismo é uma medida mais conservadora da decodificação. Esses resultados parecem indicar que para o reconhecimento de palavras no português a decodificação é a principal habilidade.

No entanto, precisamos ressaltar que, a leitura neste estudo foi medida pelo subteste do TDE que investiga o reconhecimento de palavras. Nem todas as palavras nesse subteste são morfologicamente complexas. Pode se pensar que a consciência morfológica teria um papel específico para palavras com ortografia complexa.

Em conjunto, esses resultados sugerem que a decodificação tem um papel importante no desempenho da leitura nas fases iniciais $\mathrm{da}$ escolarização e que o processamento morfológico precisa ser mais bem estudado. Precisamos apontar as limitações da metodologia empregada. No presente estudo usamos tarefas de spooneirismo e subtração de fonemas para medir o conhecimento das relações entre letra e som das palavras. Nessas tarefas as crianças tinham que manipular a relação entre letras e os fonemas das palavras. A tarefa de spooneirismo é uma tarefa mais difícil do que a tarefa de subtração de fonemas, pois envolve um grau maior de manipulação da estrutura fonêmica das palavras, por isso pode ter discriminado melhor essa habilidade entre as crianças avaliadas que já tinham mais de um ano de experiência com a leitura. $O$ índice de acerto na tarefa de subtração foi de 9 ou acima em 10 possíveis acertos. Esse resultado caracteriza um efeito de "teto" e pode não ter permitido variância suficiente para se obter correlações significativas. Porém, as correlações entre o conhecimento das correspondências entre letra e som, medidas por essa tarefa e a tarefa de spoonerismo foram significativas. Outra limitação já apontada foi a utilização do Wisc como medida de processamento fonológico. Outros estudos devem usar outras medidas desse construto para confirmar os resultados.

Apesar dessas limitações, as regressões realizadas com as tarefas de spooneirismo, de consciência morfológica e de leitura medida pelo TDE mostram que essas estão associadas de forma positiva e estatisticamente significativa e nos oferecem evidências que podem lançar luz sobre teorias da aquisição da leitura no português. Sugerem que em uma língua no meio do espectro da transparência ortográfica, as correspondências entre letra e som podem ter um papel importante para desempenhar no reconhecimento de palavras. Evidências de que o processamento no nível lexical está em funcionamento foram as correlações significativas quando medidas menos conservadoras foram usadas. Uma implicação desses resultados é a de que o processamento do som deve ser estimulado como prática pedagógica na educação infantil e no ensino fundamental. Outra, é que ressalta a importância de conhecermos melhor os processos cognitivos subjacentes ao reconhecimento de palavras e de explorarmos como esses processos se associam com outros aspectos da leitura como a compreensão de textos.

\section{Referências}

Barrera, S. \& Maluf, M. R. (2003). Consciência metalingüística e alfabetização: um estudo com crianças da primeira série do ensino fundamental. Psicologia: Reflexão e Crítica, 16(3), 491-502.

Bradley, L. \& Bryant, P. (1983). Categorizing sounds and learning to read: a causal connection. Nature, $301,419-421$.

Bruce, D. J. (1964). The analysis of word sounds. British Journal of Educational Psychology, 34, 158-170.

Bryant, P. \& Bradley, L. (1987). Problemas de leitura na criança. Porto Alegre: Artes Médicas.

Capovilla, A. \& Capovilla, F. (2000). Efeitos do treino de consciência fonológica em crianças com baixo nível sócio-econômico. Revista Psicologia: Reflexão e Crítica, 13(1), 07-24.

Cardoso-Martins, C. \& Frith, U. (1999) Consciência fonológica e habilidade de leitura na síndrome de Down. Psicologia. Reflexão e Crítica, 12(1), 209-224.

Carlisle, J. (1995). Morphological awareness and early reading achievement. Em L. Feldman (Org.) Morphological aspects of language processing_(pp. 189211). Hillsdale: Law rence Erlbaum Associates.

Carlisle, J. (2000). Awareness of the structure and meaning of morphologically complex words: impact on reading. Reading and Writing: $A n$ Interdisciplinary Journal, 12, 169-190.

Carlisle, J. \& Fleming, J. (2003). Lexical processing of morphologically complex words in the elementary years). Scientific Studies of Reading, 7(3), 239-253.

Colé, P., Marec-Breton, N., Royer, C. \& Gombert, J. E. (2003). Morphologic des mots et apprentissage de la lecture. Rééducacion Orthophonique, 213, 57-76.

Deacon, S. \& Kirby, J. (2004). Morphological awareness: just "more phonological"? the roles of morphological and phonological awareness in reading development. Applied Psycholinguistics, 25, 223-238. 
Deacon, S. \& Bryant, P. (2005). What young children do and do not know about the spelling of inflections and derivations. Developmental Science, 8(6), 583-594.

Marec-Breton, N. \& Gombert, J. (2004). A dimensão morfológica nos principais modelos de aprendizagem da leitura. Em M. R. Maluf (Org). Psicologia educacional: questões contemporâneas (pp. 105122). São Paulo: Casa do Psicólogo.

Mota, M. (2011). Consciência morfológica, aspectos cognitivos da linguagem e reconhecimento de palavras. Interação Psicológica, 15(1), 21-26.

Mota. M., Anibal, L. \& Lima, S. (2008). A morfologia derivacional contribui para a leitura e escrita no português? Psicologia: Reflexão e Crítica, 21(2), 311318.

Mota, M., Toledo, M., Bastos, R., Dias, J., Paiva, N., Mansur-Lisboa, S. F. \& Silva, D. A. (2012). Leitura Contextual e Processamento Metalinguístico no Português do Brasil: Um estudo longitudinal. Psicologia: Reflexão e Crítica, 25(2), 114-120.

Nagy, W., Berninger, V. \& Abbot, R. (2006). Contributions of morphology beyond phonology to literacy outcome of upper elementary and middle-school students. Journal of Educational Psychology, 98(1), 134-147.
Nunes, T,. Bindman, M. \& Bryant, P. (1997). Morphological strategies: developmental stages and processes. Developmental Psychology, 33(4), 637649.

Sénéchal, M., LeFevre, J., Thomas, E. \& Daley, K (1998). Reading Research Quarterly, 33(1), 96-116.

Stein, L. M. (1994). TDE - Teste de desempenho escolar: Manual para aplicação e interpretação. São Paulo: Casa do Psicólogo.

Sternberg, R.. J. (2000). Psicologia cognitiva. Porto Alegre: Artmed.

Tong, X., Deacon, S., Kirby, J., Cain, K., \& Parrila, R. (2011). Morphological awareness: a key to understanding poor reading comprehension in English. Journal of Educational Psychology, 103(3), 523-534.

Wechsler, D. (1991). WISC-III: Escala de inteligência Weschsler para crianças. São Paulo: Casa do Psicólogo.

Verhoeven, L., Schreuder, R., \& Haarman, V. (2006). Prefix identification in the reading of Dutch bysyllabic words. Reading and Writing, 19, 651-68.

Recebido em 02/10/2012

Reformulado em 14/02/2013

Aprovado em 07/03/2013

Nota dos autores:

Agradecemos às alunas de Treino de Pesquisa que ajudaram na coleta dos dados.

Sobre as autoras:

Luciene Corrêa Miranda é mestre em Psicologia pela UFJF, professora da Escola de Enfermagem da SCMJF e psicóloga Clínica.

Márcia Maria Peruzzi Elia da Mota é doutora em Psicologia pela Universidade de Oxford na Inglaterra, professora titular do programa de pós-graduação em psicologia da Universidade Salgado de Oliveira e vice-diretora do Instituto de Psicologia da UERJ. É pesquisadora do CNPq nível 2. 